STRUCTURAL BIOLOGY COMMUNICATIONS

ISSN 2053-230X

\title{
Crystal structures of the amino-terminal domain of LpoA from Escherichia coli and Haemophilus influenzae
}

\author{
Aaron Kelley, ${ }^{a}$ J. Vijayalakshmi ${ }^{\mathrm{b}}$ and Mark A. Saper ${ }^{\mathrm{a}, \mathrm{b} *}$

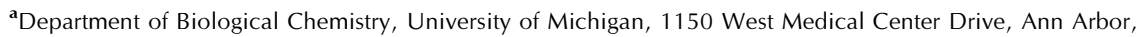 \\ MI 48109-5606, USA, and ' Program in Biophysics, University of Michigan, 930 North University Avenue, Ann Arbor, \\ MI 48109-1055, USA. *Correspondence e-mail: saper@umich.edu
}

Received 17 January 2019

Accepted 24 March 2019

Edited by S. Sheriff, Bristol-Myers Squibb, USA

Keywords: outer membrane lipoprotein; peptidoglycans; TPR domain; conformational differences; LpoA; N-terminal domains.

PDB references: amino-terminal domain of H. influenzae LpoA, 6dcj; amino-terminal domain of E. coli LpoA, $6 \mathrm{dr} 3$

Supporting information: this article has supporting information at journals.iucr.org/f

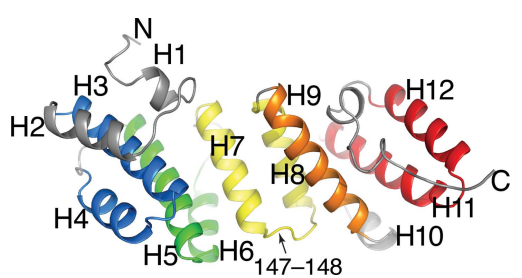

(C) 2019 International Union of Crystallography
The bacterial periplasmic protein LpoA is an outer membrane lipoprotein and an activator for the cross-linking activity of PBP1A, a bifunctional peptidoglycan synthase. Previous structures of the amino-terminal (N) domain of LpoA showed it to consist entirely of helices and loops, with at least four tetratricopeptide-like repeats. Although the previously determined orthorhombic crystal structure of the $\mathrm{N}$ domain of Haemophilus influenzae LpoA showed a typical curved structure with a concave groove, an NMR structure of the same domain from Escherichia coli was relatively flat. Here, a crystal structure of the $\mathrm{N}$ domain of $E$. coli LpoA was determined to a resolution of $2.1 \AA$ and was found to be more similar to the $H$. influenzae crystal structure than to the E. coli NMR structure. To provide a quantitative description for these comparisons, the various structures were superimposed pairwise by fitting the first half of each structure to its pairwise partner and then calculating the rotation axis that would optimally superimpose the second half. Differences in both the magnitude of the rotation and the direction of the rotation axis were observed between different pairs of structures. A $1.35 \AA$ resolution structure of a monoclinic crystal form of the $\mathrm{N}$ domain of $H$. influenzae LpoA was also determined. In this structure, the subdomains rotate $10^{\circ}$ relative to those in the original orthorhombic $H$. influenzae crystal structure to further narrow the groove between the subdomains. To accommodate this, a bound chloride ion (in place of sulfate) allowed the closer approach of a helix that forms one side of the groove.

\section{Introduction}

Bacterial cell walls are primarily comprised of peptidoglycan (PG), a polymer of disaccharides cross-linked through peptide substituents. PG provides protection against internal osmotic pressure (turgor), functions in cell division and defines cell shape. Besides the recently characterized PG polymerase RodA encoded by most bacteria (Meeske et al., 2016), Gramnegative bacteria also require a bifunctional enzyme, specifically either PBP1A or PBP1B, which can polymerize and cross-link PG and may play a role in PG repair. LpoA is an outer membrane lipoprotein that is essential for activating the cross-linking (transpeptidase) activity of PBP1A (Typas et al., 2010; Paradis-Bleau et al., 2010). LpoA is composed of two domains: a primarily helical amino-terminal $(\mathrm{N})$ domain and a carboxyl-terminal domain involved in interactions with PBP1A. Analyses of multiple crystal structures of full-length LpoA from Haemophilus influenzae (HiLpoA), together with small-angle X-ray scattering results, suggested that LpoA is a flexible molecule that is capable of extending through gaps or holes in PG in order to interact with the inner-membranebound PBP1A (Sathiyamoorthy et al., 2017). Although the 
Table 1

Expression plasmid construction.

\begin{tabular}{|c|c|c|}
\hline & EcTet $(31-252)$ & HiMon (33-253) \\
\hline Source organism & E. coli MG1655 & H. influenzae $\mathrm{Rd}$ \\
\hline DNA source & pCB40 plasmid DNA (Paradis-Bleau et al., 2010) & H. influenzae Rd genomic DNA \\
\hline Cloning method & Isothermal (NEBuilder HiFi, NEB) & Restriction enzymes \\
\hline Forward primer & agaacctgtacttcCAATCCACTCCCGATCAGTCCACTG† & CATGCCATGGCGAATTTCACGCAAACCTTACAA $\ddagger$ \\
\hline Reverse primer & $\begin{array}{l}\text { cgttatccacttccaatattttaTTTAAACGCTTTTACGTTA } \\
\text { ACCAAC } \dagger\end{array}$ & GCCGACGTCGACTTGTTGGAAATTAAGCAATGTAAG§ \\
\hline Forward primer to amplify plasmid & taaAATATTGGAAGTGGATAACGGATC & \\
\hline Reverse primer to amplify plasmid & GGATTGGAAGTACAGGTTCTCG & \\
\hline Cloning vector & pMCSG7 (Stols et al., 2002) & pETBlue-2 (Novagen) \\
\hline Expression vector & pMCSG7 & pETBlue-2 \\
\hline Expression host & E. coli Origami 2(DE3) (Novagen) & E. coli Tuner(DE3)/pLacI (Novagen) \\
\hline $\begin{array}{l}\text { Complete amino-acid sequence of } \\
\text { the construct produced }\end{array}$ & $\begin{array}{l}\text { mhhhhhhssgvdlgtenlyfqSTPDQSTAYMQGTAQADSAFY } \\
\text { LQQMQQSSDDTRINWQLLAIRALVKEGKTGQAVELFNQLP } \\
\text { QELNDAQRREKTLLAVEIKLAQKDFAGAQNLLAKITPADL } \\
\text { EQNQQARYWQAKIDASQGRPS I DLLRALIAQEPLLGAKEK } \\
\text { QQNIDATWQALSSMTQEQANTLVINADENILQGWLDLQRV } \\
\text { WEDNRNDPDMMKAGIADWQKRYPNNPGAKMLPTQLVNVKA } \\
\text { FK }\end{array}$ & $\begin{array}{l}\text { MANFTQTLQKDANASSEFYINKLGQTQELEDQQTYKLLAARV } \\
\text { LIRENKVEQSAALLRELGELNDAQKLDRALIEARISAAKN } \\
\text { ANEVAQNQLRALDLNKLSPSQKSRYYETLAIVAENRKDMI } \\
\text { EAVKARIEMDKNLTDVQRHQDNIDKTWALLRSANTGVINN } \\
\text { ASDEGNAALGGWLTLIKAYNDYIRQPVQLSQALQSWKNAY } \\
\text { PNHAAATLFPKELLTLLNFQQVEHHHHHH } \dagger \dagger\end{array}$ \\
\hline
\end{tabular}

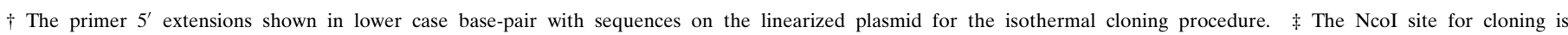

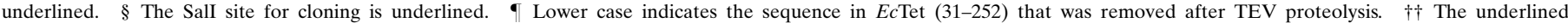
$\mathrm{N}$-terminal MA and C-terminal $\mathrm{His}_{6}$-tag residues were added to the native sequence during cloning.

interdomain linker is responsible for much of the flexibility of the molecule, twists and bends of up to $5^{\circ}$ have been observed within the $\mathrm{N}$ domain and are likely to affect the overall length of the molecule.

The structures of the $\mathrm{N}$ domain from both $E$. coli LpoA (EcLpoA) and $H i$ LpoA contain repeats of the helical tetratricopeptide-like (TPR-like) motif, which is a helix-turn-helix motif that is often involved in protein-protein interactions. Consecutive TPR-like motifs typically induce a superhelical twist to form a concave groove that is ideal for binding peptide segments of other proteins (Grove et al., 2008). For example, a conformational change of the TPR-containing protein MamA is associated with binding of its putative ligand, suggesting possible mechanisms for TPR-regulated functionality (Zeytuni et al., 2011).

The structure of the $\mathrm{N}$ domain of $E$. coli LpoA was first determined using NMR (EcNMR) and, although composed of TPR-like helix-turn-helix motifs, was observed to be relatively flat without a significant superhelical twist (Jean et al., 2014). When the orthorhombic crystal structure of the $\mathrm{N}$ domain of $H$. influenzae LpoA (HiOrt) was reported (Sathiyamoorthy et al., 2017), its subdomains 1 and 2 were noted to each be very similar to the corresponding subdomains in EcNMR. However, when the two intact N-domain structures were superimposed, based solely on least-squares fitting of their subdomains 1 (specifically residues 33-148), their subdomains 2 were related by a $45^{\circ}$ rotation (see Fig. 2 in Sathiyamoorthy et al., 2017).

To better understand the range of conformations accessible to the LpoA $\mathrm{N}$ domain, we set out to determine its structure in additional crystal environments. We determined the tetragonal crystal structure of the $\mathrm{N}$ domain of $E c \mathrm{LpoA}$ (EcTet) and found it to be closer in structure to $H i$ Ort than to $E c$ NMR. We also determined a $1.35 \AA$ resolution structure of the $\mathrm{N}$ domain of $H i$ LpoA in a monoclinic space group; inspection of this structure showed it to have a more concave cleft than the previously reported orthorhombic form (PDB entry 4p29; Sathiyamoorthy et al., 2017).

\section{Materials and methods}

\subsection{Macromolecule production}

To express the $E c \mathrm{LpoA} \mathrm{N}$ domain, the gene corresponding to residues 31-252 of EcLpoA was amplified using PCR from genomic DNA (E. coli strain MG1655) and cloned into pMCSG7 (Stols et al., 2002). The primers used to amplify the domain from $E c$ LpoA and to linearize the pMCSG7 plasmid are shown in Table 1 . The resulting construct contained an $\mathrm{N}$-terminal $\mathrm{His}_{6}$ tag and TEV protease cleavage site (detailed in Table 1). The construct was confirmed by carrying out Sanger sequencing in both the forward and reverse directions.

The pMCSG7-EcLpoA-N plasmid was transformed into E. coli Origami 2(DE3) cells (Novagen). The cells were grown in two 2.81 flasks, each containing $450 \mathrm{ml}$ Terrific Broth. After inoculation and initial growth, the flasks were transferred to an incubator at $22^{\circ} \mathrm{C}$ and IPTG was added to $0.45 \mathrm{mM}$ for induction. The cells were harvested $16 \mathrm{~h}$ after induction by centrifugation at $5900 \mathrm{~g}$, after which they were resuspended with DNase and protease-inhibitor tablets and frozen at $-80^{\circ} \mathrm{C}$. The cells were lysed via three passes through an Emulsiflex-C3 homogenizer at $4^{\circ} \mathrm{C}$. The clarified lysate was applied onto a $5 \mathrm{ml}$ column packed with HisPur Cobalt Resin (Thermo Fisher), washed and then eluted with $180 \mathrm{mM}$ imidazole. The protein was treated with TEV protease overnight at $4^{\circ} \mathrm{C}$ and then passed through a HisPur Ni-NTA Resin column (Thermo Fisher) to remove cleaved His ${ }_{6}$ tag and TEV protease. Concentrated protein $\left(25 \mathrm{mg} \mathrm{ml}^{-1}\right)$ was frozen in aliquots containing $10 \%$ glycerol. Before crystallization trials, the aliquots were passed through a gel-filtration column (Superdex HR 75 10/300) to remove glycerol. The predicted sequence of the mature $E c \mathrm{LpoA} \mathrm{N}$-domain protein was serine 
Table 2

Crystallization.

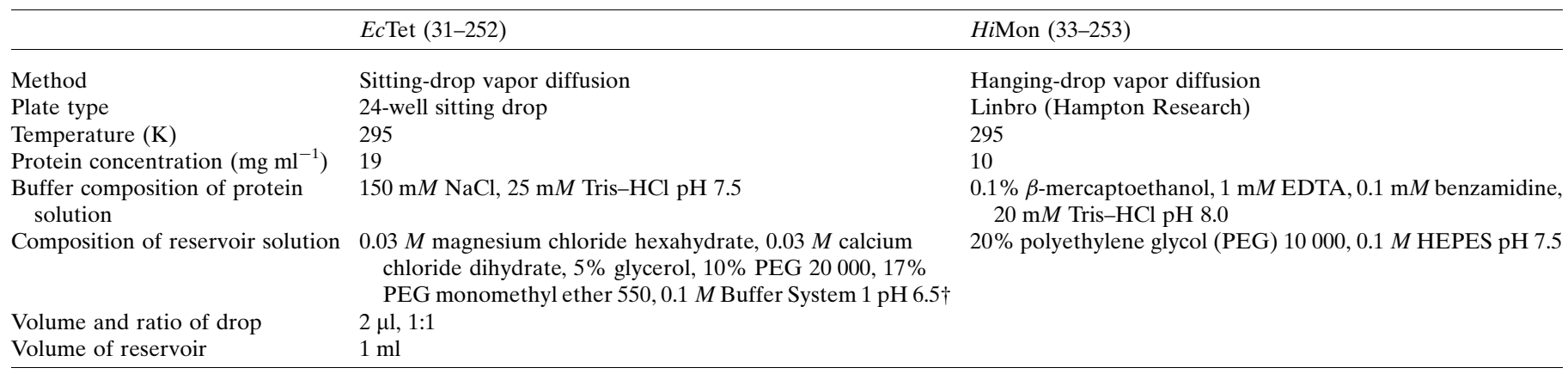

$\dagger$ Buffer System 1, 1.0 M stock solution: $30 \mathrm{ml} 1 M$ 2-( $N$-morpholino)ethanesulfonic acid (MES) $\mathrm{pH} 3.1$ was titrated with $24.1 \mathrm{ml} 1 M$ imidazole $\mathrm{pH} 10.2$ to a final $\mathrm{pH}$ of 6.5 .

followed by residues 31-252 of EcLpoA (formula weight 25 154).

The cloning, expression and purification of residues 33-253 of the HiLpoA N domain were identical to the procedures described previously (Sathiyamoorthy et al., 2017). The gene fragment with NcoI and Sall restriction sites corresponding to residues 33-253 of $H i$ LpoA was amplified using PCR from genomic DNA isolated from the $H$. influenzae $\mathrm{Rd}$ strain (ATCC catalog No. 9008). After digestion, the purified fragment was ligated into the pETBlue-2 (Novagen) plasmid. The plasmid encoding the $\mathrm{N}$-terminal residues $\mathrm{MA}$ and the C-terminal $\mathrm{His}_{6}$ tag was transformed into E. coli Tuner(DE3)/ pLacI (Novagen) cells for expression. The formula weight of the purified protein was predicted to be 26224 . Details of the construct are shown in Table 1.

\subsection{Crystallization}

Initial crystals of the $\mathrm{N}$ domain of EcLpoA grew from condition A1 of the Morpheus screen (Molecular Dimensions). The $\mathrm{N}$ domain of $H i \mathrm{LpoA}$ was previously reported to form orthorhombic crystals (Sathiyamoorthy et al., 2017). In the current work, we grew monoclinic crystals of the $\mathrm{N}$ domain of $H i$ LpoA from different conditions. Crystals were harvested into precipitant containing $10 \%$ glycerol and were immediately cooled in liquid nitrogen. Crystallization information is given in Table 2.

\subsection{Data collection and processing}

Diffraction data were collected from the tetragonal crystals of the $\mathrm{N}$ domain of $E c$ LpoA ( $E c$ Tet) on the LS-CAT beamline 21-ID-D at the Advanced Photon Source (APS), Argonne National Laboratory and were processed with $H K L-2000$ (Otwinowski \& Minor, 1997). Diffraction data for the monoclinic crystals of the $\mathrm{N}$ domain of $H i$ LpoA (HiMon) were collected on the DND-CAT beamline 5-ID-B at APS and were processed with $d^{*} T R E K$ (Pflugrath, 1999). Details of data collection and processing are presented in Table 3.

\subsection{Structure solution and refinement}

Initial attempts to solve the structure of EcTet by molecular replacement in Phaser (McCoy et al., 2007; Adams et al., 2010) with search models containing each subdomain of $E c \mathrm{NMR}$ (PDB entry 2mhk; Jean et al., 2014) were unsuccessful. The two subdomains of $\mathrm{HiOrt}$ ( $29 \%$ sequence identity to EcTet), after pruning side chains that were not common with EcTet in Sculptor (Bunkóczi \& Read, 2011), were used as search models. Molecular-replacement calculations in Phaser resulted in a log-likelihood gain (LLG) of 312 and a translationfunction $Z$-score (TFZ) of 17.1, and confirmed that the space group for these data was $P 4_{3} 2_{1} 2$. The structure was manually built with Coot (Emsley et al., 2010) and refined in PHENIX (v.1.12; Adams et al., 2010) with data to $2.1 \AA$ resolution (99\% complete) and three TLS domains to a final $R_{\text {work }}$ and $R_{\text {free }}$ of 0.177 and 0.213 , respectively. Waters were added and validated in Coot. Simulated-annealing OMIT maps were consulted to minimize the bias from the starting model (Supplementary Fig. S1). Details of the refinement and model validation are summarized in Table 4. The coordinates and structure factors were deposited in the PDB as entry 6dr3. Residue numbering corresponds to that of GenBank sequence NP_417616.1 and not to the sequence numbering for EcNMR (PDB entry 2mhk).

In solving the $H i$ Mon structure, molecular-replacement calculations with Phaser using the intact structure of $\mathrm{HiOrt}$ (PDB entry 4p29) as a search model (Sathiyamoorthy et al., 2017) were unsuccessful. Breaking the search model into two subdomains as above successfully placed two copies of each of the two subdomains in the asymmetric unit, consistent with two monomers. The LLG and TFZ values were 2681 and 33.3, respectively, for the molecular-replacement solution. As above, the HiMon structure was modified in Coot and refined with PHENIX with data to $1.35 \AA$ resolution ( $90 \%$ complete) and anisotropic $B$ factors to a final $R_{\text {work }}$ and $R_{\text {free }}$ of 0.155 and 0.190 , respectively. TLS refinement was not performed as it was redundant with the anisotropic $B$ factors. Hydrogens were included in their riding positions. Details of the refinement and model validation are summarized in Table 4 and an OMIT map is shown in Supplementary Fig. S2. Coordinates and structure factors were deposited in the PDB as entry $6 \mathrm{dcj}$.

\subsection{Structure comparisons}

All structural superimpositions were performed by leastsquares fitting using the super routine of PyMOL (v.2.2; 
Table 3

Data collection and processing.

Values in parentheses are for the outer shell.

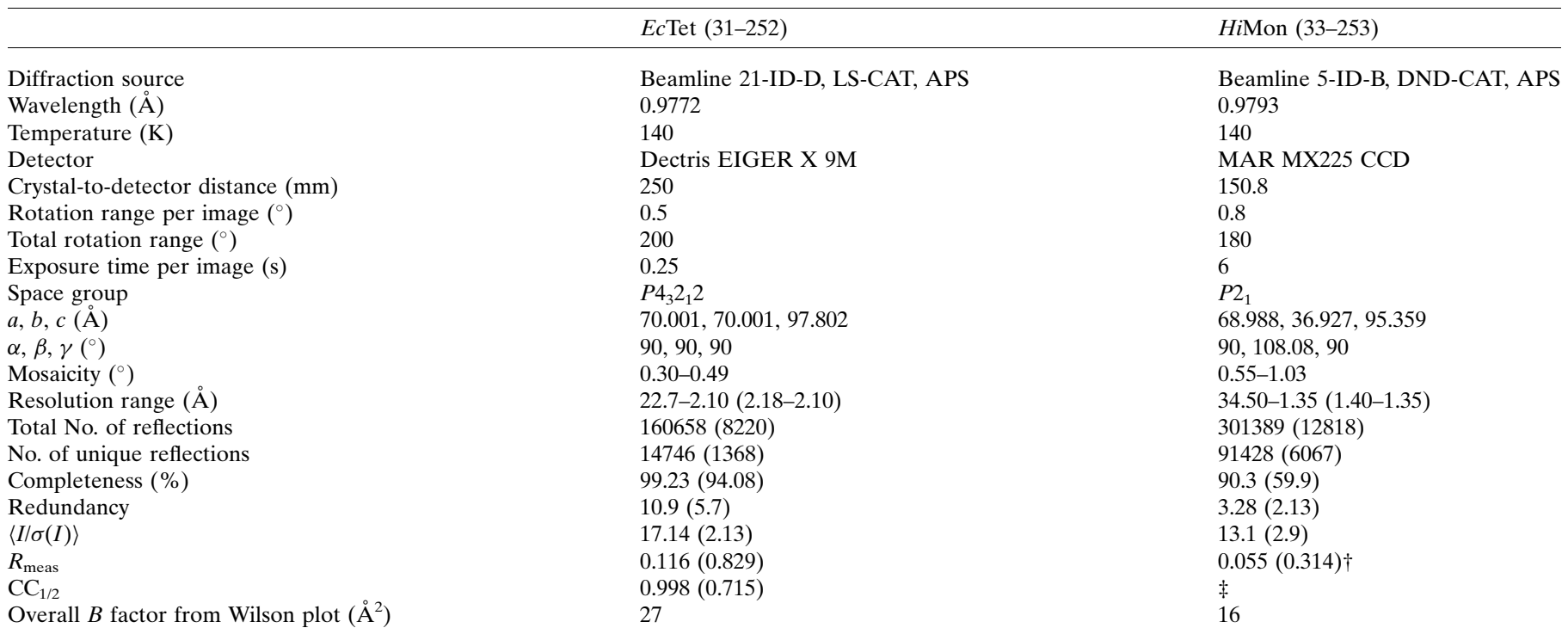

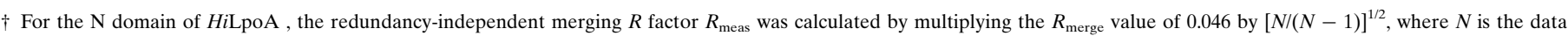
redundancy. $\ddagger$ Not available.

Table 4

Structure solution and refinement.

Values in parentheses are for the outer shell.

\begin{tabular}{|c|c|c|}
\hline & $E c$ Tet (31-252) & HiMon (33-253) \\
\hline PDB entry & $6 \mathrm{dr} 3$ & $6 \mathrm{dcj}$ \\
\hline Resolution range $(\AA)$ & $22.7-2.10(2.18-2.10)$ & $34.44-1.35(1.40-1.35)$ \\
\hline Completeness (\%) & $99.23(94.08)$ & $90.52(60.70)$ \\
\hline$\sigma$ Cutoff & None & None \\
\hline No. of reflections, working set & $13194(1230)$ & $91406(6065)$ \\
\hline No. of reflections, test set & 1465 (137) & $7328(490)$ \\
\hline Final $R_{\text {cryst }}$ & $0.177(0.249)$ & $0.155(0.228)$ \\
\hline Final $R_{\text {free }}$ & $0.213(0.280)$ & $0.190(0.279)$ \\
\hline \multicolumn{3}{|l|}{ No. of non-H atoms } \\
\hline Protein & 1776 & 3652 \\
\hline Ion & 0 & 2 \\
\hline Water & 151 & 714 \\
\hline Total & 1927 & 4368 \\
\hline \multicolumn{3}{|l|}{ R.m.s. deviations } \\
\hline Bonds $(\AA ̊)$ & 0.002 & 0.007 \\
\hline Angles $\left(^{\circ}\right)$ & 0.44 & 1.17 \\
\hline \multicolumn{3}{|l|}{ Average $B$ factors $\left(\AA^{2}\right)$} \\
\hline Overall & 38 & $25 \dagger$ \\
\hline Protein & 38 & 22 \\
\hline Ion & n.a. & 21 \\
\hline Water & 40 & 35 \\
\hline \multicolumn{3}{|l|}{ Ramachandran plot } \\
\hline Most favored (\%) & 97.29 & 99.54 \\
\hline Allowed (\%) & 2.71 & 0.46 \\
\hline Rotamer outliers (\%) & 0.53 & 0.51 \\
\hline MolProbity score/clashscore & $1.01 / 1.41$ & $1.03 / 2.46$ \\
\hline
\end{tabular}

$\dagger$ The $H i$ Mon structure was refined with anisotropic $B$ factors. Isotropic equivalent values are shown in this table.

Schrödinger). This routine in PyMOL iteratively rejects paired residues if the interatomic distance exceeds two times the overall r.m.s. deviation. Object transformation matrices from super were converted into an equivalent angular rotation about a screw axis (GEM; E. Fauman, unpublished work), which was displayed as a cylindrical arrow using a compiled graphics object in $P y M O L$. To ascertain the difference in curvature (the relative difference in subdomain 2 rotation) between two LpoA N-domain structures, subdomains 1 of the structures were first superimposed. Subdomains 2 were then superimposed and the matrix from this latter transformation was converted into an equivalent rotation as above. All figures were prepared in $P y M O L$.

\section{Results}

\subsection{Comparison of ECTet and ECNMR (PDB entry 2mhk)}

As described in Section 2, the protein that was crystallized to form EcTet was designed to include Ser30 followed by residues 31-252 of EcLpoA. Two subdomains consisting of 13 helices and the loops between them were observed in EcTet, with subdomain 1 consisting of helices $\mathrm{H} 1-\mathrm{H} 7$ and spanning residues 30-147 and subdomain 2 consisting of helices H8H13 and spanning residues 148-252 (Fig. 1). Residues 242-247 form one turn of an $\alpha$-helix, while two turns were observed in both $E c$ NMR and $H i$ Ort. Only weak density was observed corresponding to residues 247-252, indicating that these residues were likely to be partially disordered.

Subdomains 1 and 2 of $E c$ Tet were individually superimposed on the respective subdomains of $E c \mathrm{NMR}$, and analysis of this superposition confirmed similar local folds (subdomain 1, r.m.s.d. on $\mathrm{C}^{\alpha}$ atoms of $2.0 \AA$; subdomain 2, r.m.s.d. on $\mathrm{C}^{\alpha}$ atoms of $1.5 \AA$; see Table 5). However, superimposing the full-length $\mathrm{N}$ domains showed quite a large difference in backbone structure (r.m.s.d. of $3.5 \AA$ ), with this difference explained by different rotations between the two 
subdomains in the two structures. The difference in domain curvature (calculated as described in Section 2) showed that $E c$ NMR subdomain 2 required a $33^{\circ}$ rotation around the axis displayed in Fig. 2 in order to optimally superimpose it onto $E c$ Tet. That is, in contrast to $E c \mathrm{NMR}, E c$ Tet was observed to exhibit more of a superhelical twist and a more pronounced concave groove between the two subdomains.
3.2. Comparison of the overall conformations of EcTet and HiOrt

After aligning subdomain 1 of the EcTet crystal structure onto that of $\mathrm{HiOrt}$, subdomain 2 of EcTet must be rotated by $17.4^{\circ}$ around the axis shown in Fig. 3 to superimpose it on subdomain 2 of $H i$ Ort. That is, the EcTet concave curvature, while more pronounced than that for EcNMR, was observed

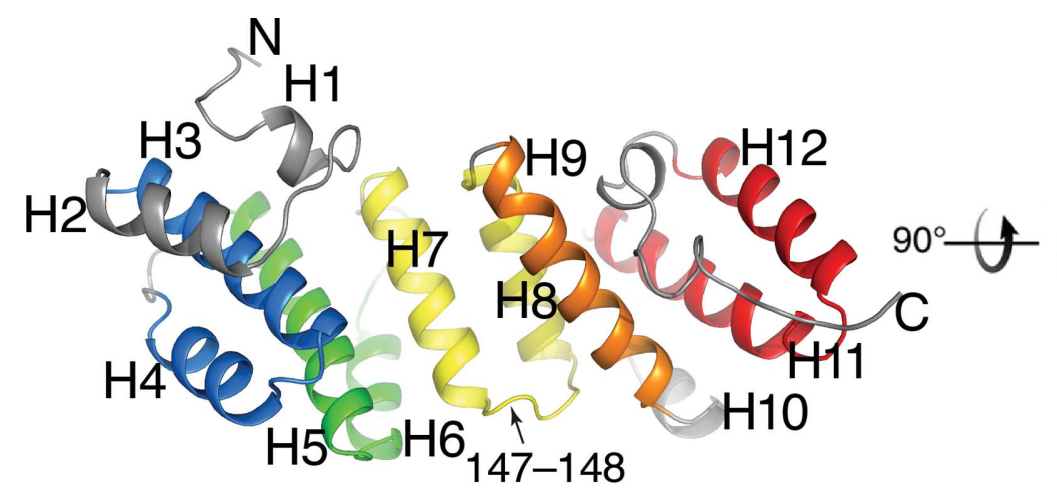

Figure 1

Cartoon representation of the EcTet domain, highlighting the TPR-like motifs in different colors. Left, top view looking into the groove located above helix H7. Right, side view showing the concave shape of the domain and the locations of the subdomains.

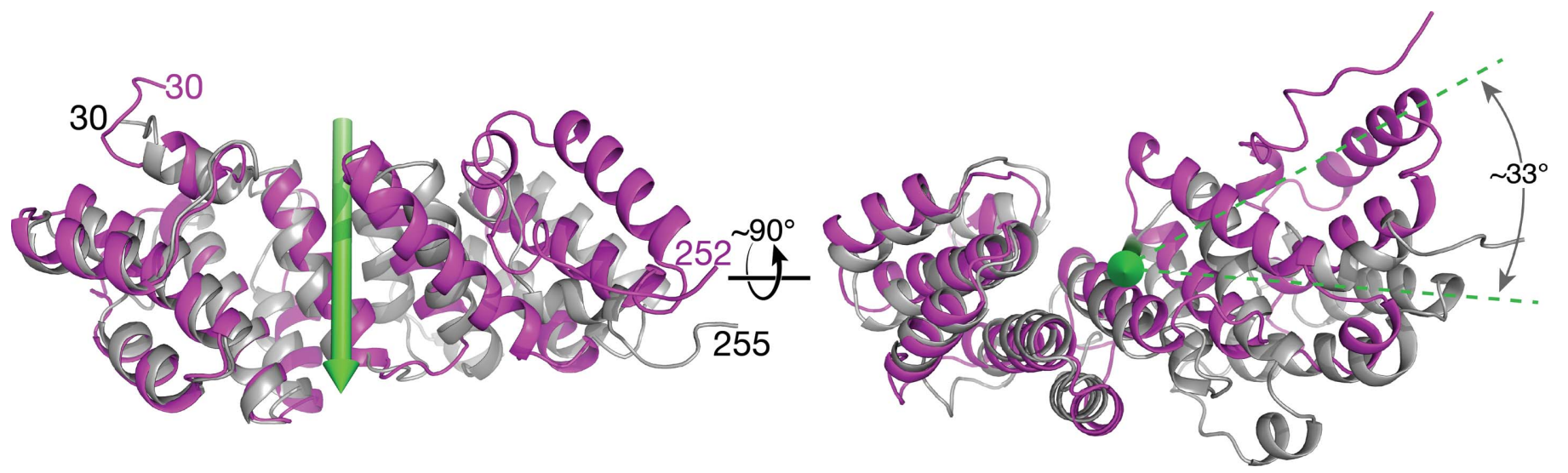

Figure 2

Superimposition of the $E c$ NMR structure (gray) and the EcTet crystal structure (magenta) accomplished by fitting their subdomains 1 . The green cylindrical arrow indicates the $33^{\circ}$ rotation axis relating the subdomains 2 of the two superimposed structures. Right, side view showing the rotation axis end-on.
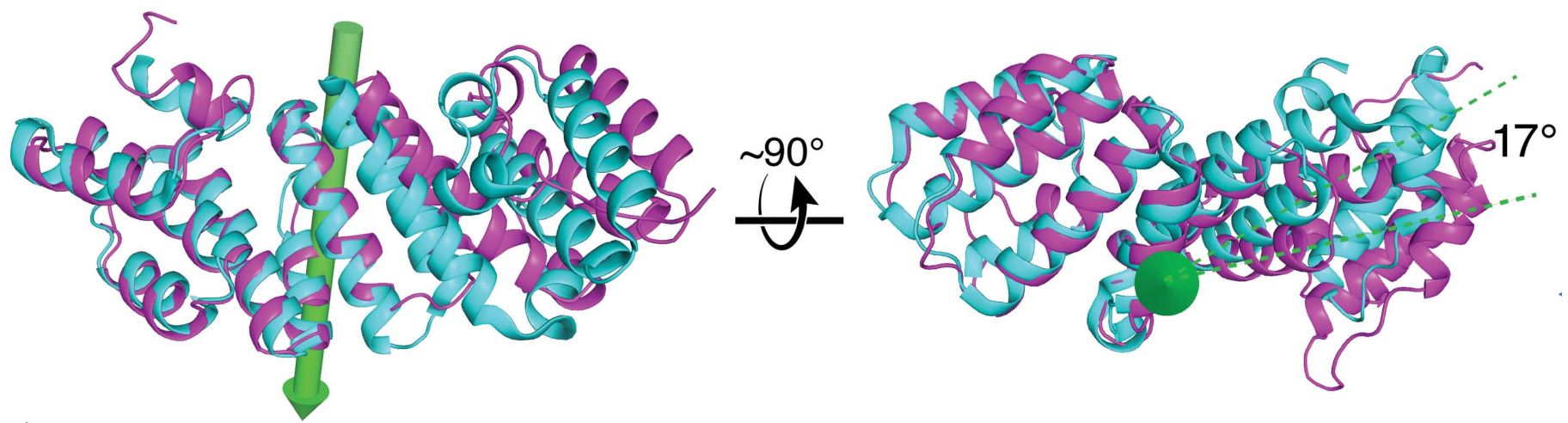

Figure 3

Superimposition of $H i$ Ort (cyan) and $E c$ Tet (magenta) accomplished by fitting their subdomains 1 . Right, side view showing the $17^{\circ}$ rotation between the respective subdomains 2 . 
Table 5

Summary of structural comparisons of LpoA N domains.

\begin{tabular}{lllllr}
\hline & R.m.s.d. $\dagger(\AA)$ & & & \\
\cline { 2 - 5 } Aligned structures & Overall & Subdomain 1 & Subdomain 2 & & Rotation $\ddagger\left({ }^{\circ}\right)$ \\
\hline$E c$ NMR $/ E c$ Tet & $3.7(209 / 222)$ & $2.0(112 / 118)$ & $1.5(94 / 105)$ & 33 & Translation§ $(\AA)$ \\
$H i$ Ort $/ E c$ Tet & $2.4(199 / 221)$ & $0.9(82 / 118)$ & $0.8(77 / 104)$ & 17 & 0.4 \\
$H i$ Mon $/ E c$ Tet & $3.2(206 / 221)$ & $1.0(99 / 116)$ & $1.2(77 / 104)$ & 27 & -1.0 \\
$H i$ Ort $/ H i$ Mon & $1.0(207 / 221)$ & $0.3(96 / 116)$ & $0.4(80 / 105)$ & 10 & -0.5 \\
\hline
\end{tabular}

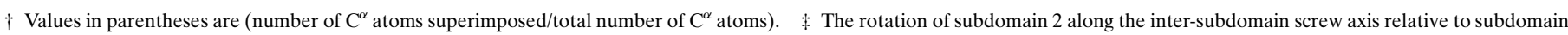
1. $\S$ The translation along the inter-subdomain screw axis.

to be less prominent than that of $\mathrm{HiOrt}$. A similar comparison with the high-resolution $\mathrm{HiMon}$ structure (see Section 3.3 below) required a $27^{\circ}$ rotation around a similar axis in order for $E c$ Tet subdomain 2 to best superimpose on that of HiMon (Table 5).

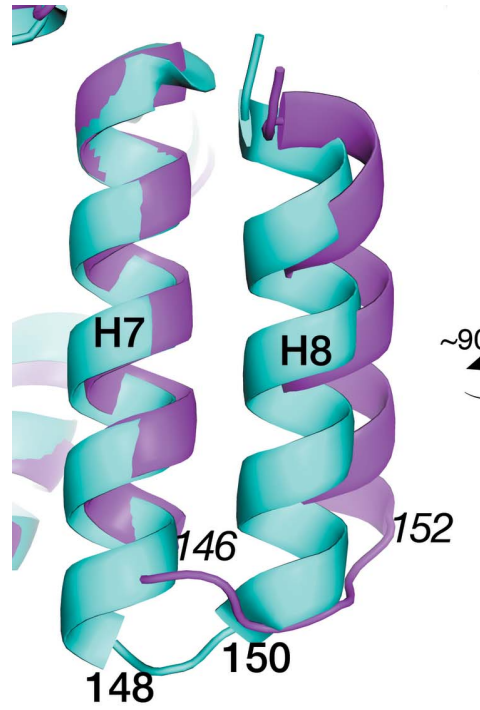

(a)

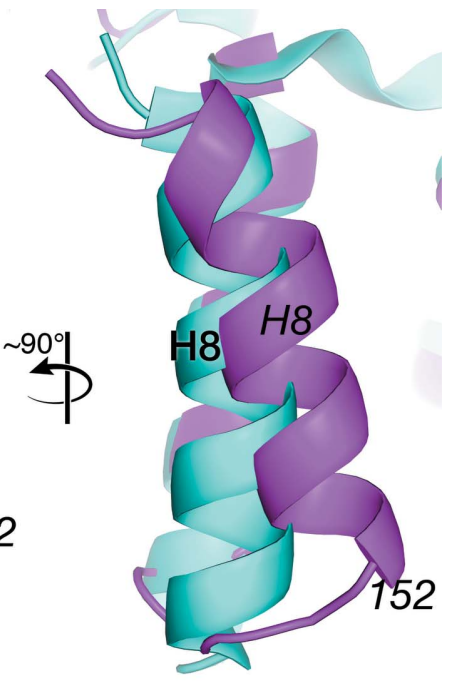

(b)
Figure 4

Helices $\mathrm{H} 7$ and $\mathrm{H} 8$ from both $\mathrm{HiOrt}$ (cyan) and $\mathrm{EcTet}$ (magenta) after fitting their subdomains 1 . A front view $(a)$ and side view $(b)$ show $\mathrm{H} 7$ and $\mathrm{H} 8$ to be mostly parallel in $\mathrm{HiOrt}$, but $\mathrm{H} 8$ is rotated by about $25^{\circ}$ in $E c$ Tet, requiring a longer loop (residues 146-152) between the two helices.
The differences between the overall conformations of EcTet and $\mathrm{HiOrt}$ coincided with, and may have originated from, differences between the junctions of their two subdomains, specifically the loop between helices $\mathrm{H} 7$ and $\mathrm{H} 8$. Inspection of HiOrt (cyan) showed its $\mathrm{H} 7$ and $\mathrm{H} 8$ helical axes to be parallel to each other and the loop between the helices (residues 148RKD-150) to be relatively short (Fig. 4a). Inspection of the EcTet structure (magenta) showed its H8 helix to make an angle of approximately $25^{\circ}$ with $\mathrm{H} 7$ and the loop between them (residues 146-SQGRPSI-152) to be longer (Fig. 4b). The inspection also showed its $\mathrm{H} 7$ helix to be about one turn shorter than $\mathrm{H} 7$ in $\mathrm{HiOrt}$. The change in conformation is not explained by the differences in the loop sequence. The difference between the interhelix angles of $E c$ Tet and $H i O r t$ appeared to be consistent with the different rotational relationships between their two subdomains.

\subsection{Comparison of HiMon with HiOrt}

As for HiOrt (PDB entry 4p29; Sathiyamoorthy et al., 2017), the $H i$ Mon crystal structure was also found to contain two molecules per asymmetric unit. In $H i$ Mon, molecules $A$ and $B$ superimposed with an r.m.s.d. of $0.3 \AA$ for $194 \mathrm{C}^{\alpha}$ atoms of a total of 211 residues in each molecule. HiMon molecules $A$ and $B$ were calculated to be related by a local $176^{\circ}$ rotation axis and a $12.2 \AA$ translation along an axis parallel to, but not coincident with, the crystallographic $b$ axis. Moreover, $\beta$-turn 189-192 appeared to be better ordered in molecule $A$ than in molecule $B$, which is likely to be because of the crystal-packing
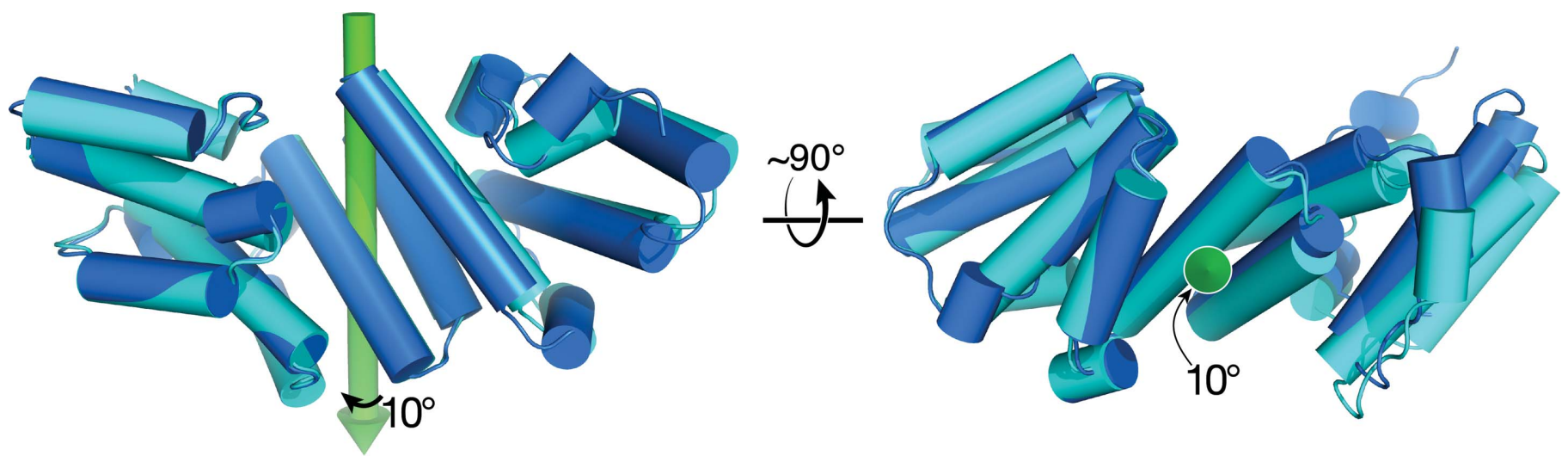

Figure 5

Superimposition of $H i$ Mon (blue) on the previously reported $H i$ Ort structure (cyan) accomplished by fitting their subdomains 1 , and showing the $10^{\circ}$ rotation axis necessary to then optimally superimpose their subdomains 2 . 


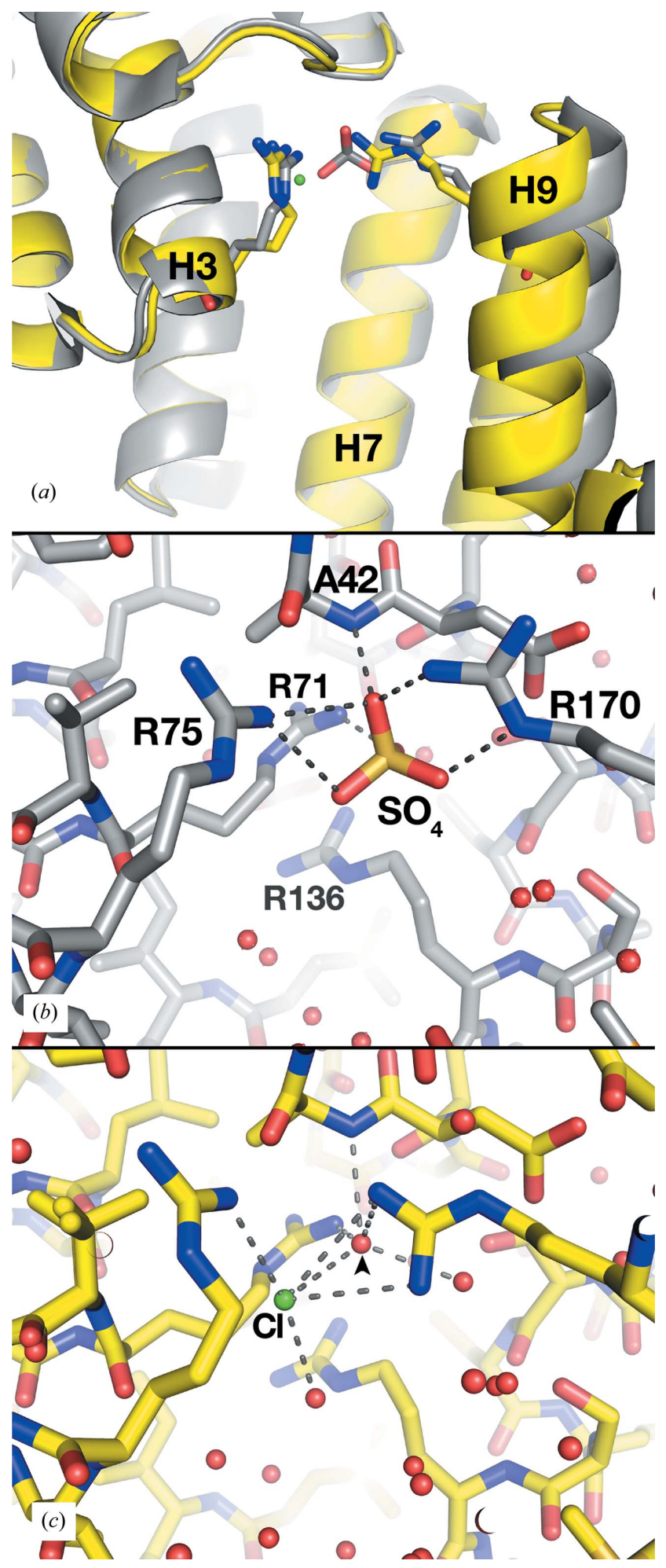

Figure 6

Superimposition of $H i$ Mon (yellow) on $H i$ Ort (gray) suggesting that their binding of different anions can accommodate the different widths of their grooves. (a) Both structures are shown with the location of the sulfate bound in the groove of $\mathrm{HiOrt}$ and the chloride (green sphere) in $\mathrm{HiMon}$. (b) Close-up view of sulfate $\left(\mathrm{SO}_{4}\right)$ ligated to arginine residues 75,170 and 71 of $\mathrm{HiOrt}$ (c) Close-up view of chloride (Cl) bound in the HiMon structure. Chloride was modeled as the bound anion in $\mathrm{HiMon}$ because the density was significantly larger than a water molecule, chloride was the only anion present in crystallization solutions and the distances to neighboring atoms were $3.2-3.4 \AA$. environment. No interpretable electron density corresponding to residue 216 of either molecule was observed, and hence this residue was omitted from the deposited PDB coordinates.

Since the $A$ and $B$ molecules were essentially identical in both $\mathrm{HiOrt}$ and $\mathrm{HiMon}$, the respective $B$ chains were arbitrarily chosen for comparison here. When subdomains 1 (116 residues, 33-148) from each crystal structure were compared, $96 \mathrm{C}^{\alpha}$ atoms were superimposed with an r.m.s.d. of $0.3 \AA$. Similarly, for subdomain 2 (149-248, 105 residues) $80 \mathrm{C}^{\alpha}$ atoms were superimposed with an r.m.s.d. of $0.4 \AA$. Comparing the relative curvatures of the two structures showed that the $\mathrm{HiOrt}$ subdomain 2 must be rotated by $10^{\circ}$ (with an $0.5 \AA$ translation) about an axis passing approximately through the center of the structure to become well superimposed on subdomain 2 of $\mathrm{HiMon}$ (Fig. 5). This rotation coincided with an increase in the curvature of the domain and a narrowing of the concave cleft.

Previously, we described a positively charged pocket that was underneath helix $\mathrm{H} 3$ of $\mathrm{HiOrt}$ but was accessible from the concave cleft (Sathiyamoorthy et al., 2017). In that structure we identified a sulfate ion $\left(\mathrm{SO}_{4}\right)$ ligated by three arginine side chains (Arg71, Arg75 and Arg170) and a main-chain amide (Ala42) (Fig. 6b). In the HiMon crystals, which were grown in the absence of sulfate, a chloride ion $(\mathrm{Cl}$ in Fig. $6 c)$ bound instead of sulfate, but was about $2.5 \AA$ closer to Arg75, which assumed a different rotamer. A water molecule (black arrow in Fig. 6c) occupied the position of the former sulfate. Also, Arg170 took on a different conformation, which allowed a closer approach to the chloride. This movement of Arg170 coincided with the movement of helix H9 of subdomain 2 closer to $\mathrm{H} 3$ of subdomain 1 by $1-2 \AA$ (Fig. $6 a$ ), which in turn narrowed the concave cleft formed by helices H3 and H9 above helix H7. These observations suggested that the binding of a natural ligand in the cleft of the $\mathrm{N}$ domain could affect the overall curvature of the domain.

\section{Discussion}

The comparison of $E c \mathrm{NMR}$ and $H i$ Ort was previously discussed by Sathiyamoorthy et al. (2017). Superimposing subdomains 1 or subdomains 2 of each molecule individually showed each of them to have similar $\mathrm{C}^{\alpha}$ structures in the two molecules (r.m.s.d. values of 1.6 and $1.7 \AA$, respectively). However, superimposing the intact molecules based on fitting their subdomains 1 required a substantial additional rotation $\left(45^{\circ}\right)$ of subdomain 2 of one molecule to superimpose it optimally on subdomain 2 of the other molecule. In other words, the relationship between subdomains 1 and 2 in $E c$ NMR was observed to be quite different from this relationship in $H i$ Ort, which is reflective of $E c$ NMR being relatively flat and lacking the superhelical twist observed in $\mathrm{HiOrt}$.

To help judge whether the flat conformation of the $\mathrm{N}$ domain of $E c \mathrm{LpoA}$ in $E c \mathrm{NMR}$ was a necessary consequence of its amino-acid sequence or was rather, at least in part, a consequence of the solution conditions used, we also analyzed the EcTet crystal structure. The EcTet crystal structure showed more of a curved than a flat conformation, indicating that the 


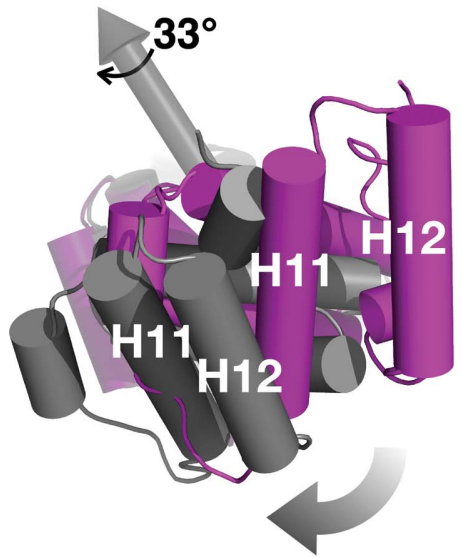

(a)

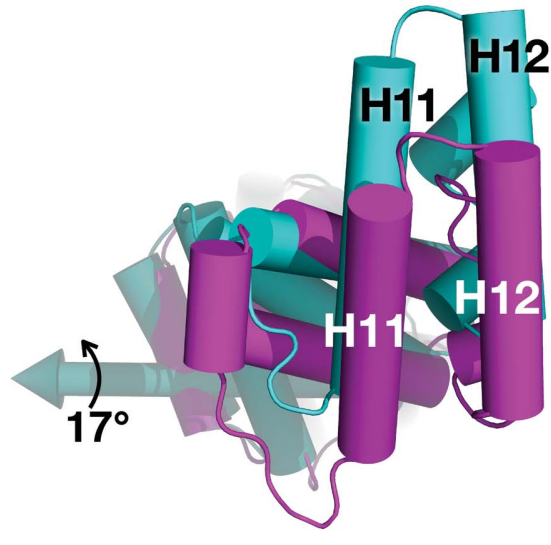

(b)

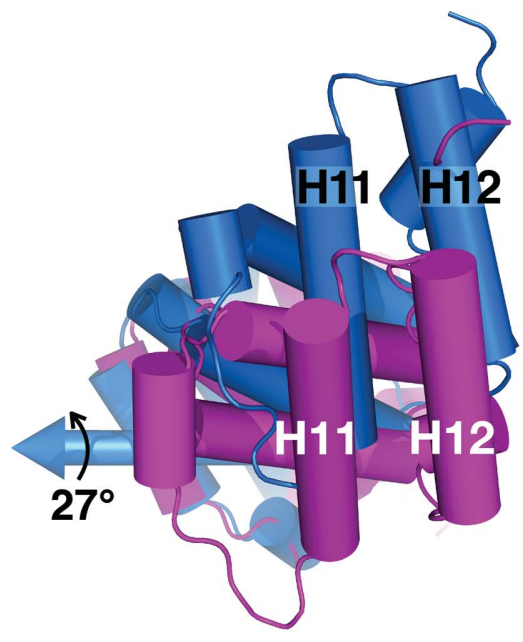

(c)

Figure 7

End-on views of structures superimposed on EcTet (magenta). Also displayed are the rotation axes relating subdomains 2 between $E c$ Tet and the (a) EcNMR (gray), (b) HiOrt (cyan) and (c) HiMon (blue) structures. The orientations of these axes in the HiOrt and $H i$ Mon crystal structures were found to be similar to each other but significantly different from that in EcNMR.

flat conformation of the EcLpoA $\mathrm{N}$ domain observed in the $E c$ NMR solution structure was not mandated by its sequence. Based on the calculated rotation angles to superimpose subdomains 2, the EcTet crystal structure showed a subdomain 2 orientation intermediate between those of $\mathrm{HiOrt}$ and $\mathrm{EcNMR}$. Judging from just these measurements, EcTet in the crystalline environment appeared to be closer in structure to $\mathrm{HiOrt}$ than to EcNMR. However, as shown in Fig. 7, the rotation axis relating subdomains 2 of $E c \mathrm{NMR}$ and $E c$ Tet was significantly different from that between $\mathrm{HiOrt}$ (or HiMon) and EcTet. To further compare the three structures, we measured an interatomic distance between helices $\mathrm{H} 3$ and $\mathrm{H} 9$ as an indicator of the width of the groove. Despite the large rotation angle relating subdomains 2 of $E c$ Tet and $E c$ NMR, the two structures displayed similar groove widths of 19.5 and $19.7 \AA$, respectively (not shown; based on the distance between $\mathrm{C}^{\alpha}$ of Lys75 and $\mathrm{C}^{\alpha}$ of Ala176), while $\mathrm{HiOrt}$ displayed a narrower groove with a width of only $15.0 \AA$ (measured between the structurally homologous residues Arg75 and Lys177). The similarity of the groove widths in the EcNMR and EcTet structures might be explained by the orientation of the rotation axis relating their subdomains 2 (Fig. 7a) and its difference from the axis relating subdomains 2 of $\mathrm{HiOrt}$ and $\mathrm{EcT}$ Tet and from the axis relating subdomains 2 of $H i$ Mon and $E c$ Tet (Figs. $7 b$ and $7 c$ ). The effect of the difference can also be observed by the relative positions of $\mathrm{H} 11$ and $\mathrm{H} 12$ in the two pairs of structures (compare Figs. $7 a$ and 7b). Analysis of the high-resolution HiMon structure demonstrated that the domain can assume a more concave structure than previously observed in $\mathrm{HiOrt}$. The groove of $\mathrm{HiMon}$ as defined above was measured to be $1.8 \AA$ narrower than the $H i$ Ort groove.

All of the crystal structures presented here showed a more curved LpoA $\mathrm{N}$ domain than was observed in the EcNMR structure. These crystal and NMR structures taken together clearly indicate the $\mathrm{N}$ domain to be inherently flexible.
Crystal-packing forces may have preferentially selected a more compact structure, but in solution the protein dynamics were less constrained, allowing the molecule to sample other conformations. The intrinsic flexibility of the $\mathrm{N}$ domain would be expected to affect the location of the $\mathrm{C}$ domain in the intact LpoA structure and its ability to interact with the PBP1A synthase transpeptidase domain in the periplasm, as discussed previously (Sathiyamoorthy et al., 2017).

\section{Acknowledgements}

The authors thank Dr Hanseong Kim and Professor Uhn-Soo Cho for help with data collection from the EcTet crystal and Jerry Brown for his editorial expertise. We thank the outstanding synchrotron support staff at LS-CAT and DNDCAT. This research used resources of the Advanced Photon Source (APS), a US Department of Energy (DOE) Office of Science User Facility operated for the DOE Office of Science by Argonne National Laboratory under Contract No. DEAC02-06CH11357. LS-CAT Sector 21 was supported by the Michigan Economic Development Corporation and the Michigan Technology Tri-Corridor (grant 085P1000817). The HiMon data were acquired at the DuPont-NorthwesternDow Collaborative Access Team (DND-CAT) located at Sector 5 of the APS. DND-CAT is supported by E. I. DuPont de Nemours \& Co., The Dow Chemical Company and the State of Illinois.

\section{References}

Adams, P. D., Afonine, P. V., Bunkóczi, G., Chen, V. B., Davis, I. W., Echols, N., Headd, J. J., Hung, L.-W., Kapral, G. J., GrosseKunstleve, R. W., McCoy, A. J., Moriarty, N. W., Oeffner, R., Read, R. J., Richardson, D. C., Richardson, J. S., Terwilliger, T. C. \& Zwart, P. H. (2010). Acta Cryst. D66, 213-221.

Bunkóczi, G. \& Read, R. J. (2011). Acta Cryst. D67, 303-312. 
Emsley, P., Lohkamp, B., Scott, W. G. \& Cowtan, K. (2010). Acta Cryst. D66, 486-501.

Grove, T. Z., Cortajarena, A. L. \& Regan, L. (2008). Curr. Opin. Struct. Biol. 18, 507-515.

Jean, N. L., Bougault, C. M., Lodge, A., Derouaux, A., Callens, G., Egan, A. J. F., Ayala, I., Lewis, R. J., Vollmer, W. \& Simorre, J.-P. (2014). Structure, 22, 1047-1054.

McCoy, A. J., Grosse-Kunstleve, R. W., Adams, P. D., Winn, M. D., Storoni, L. C. \& Read, R. J. (2007). J. Appl. Cryst. 40, 658-674.

Meeske, A. J., Riley, E. P., Robins, W. P., Uehara, T., Mekalanos, J. J., Kahne, D., Walker, S., Kruse, A. C., Bernhardt, T. G. \& Rudner, D. Z. (2016). Nature (London), 537, 634-638.

Otwinowski, Z. \& Minor, W. (1997). Methods Enzymol. 276, 307326.
Paradis-Bleau, C., Markovski, M., Uehara, T., Lupoli, T. J., Walker, S., Kahne, D. E. \& Bernhardt, T. G. (2010). Cell, 143, 1110-1120.

Pflugrath, J. W. (1999). Acta Cryst. D55, 1718-1725.

Sathiyamoorthy, K., Vijayalakshmi, J., Tirupati, B., Fan, L. \& Saper, M. A. (2017). J. Biol. Chem. 292, 17626-17642.

Stols, L., Gu, M., Dieckman, L., Raffen, R., Collart, F. R. \& Donnelly, M. I. (2002). Protein Expr. Purif. 25, 8-15.

Typas, A., Banzhaf, M., van den Berg van Saparoea, B., Verheul, J., Biboy, J., Nichols, R. J., Zietek, M., Beilharz, K., Kannenberg, K., von Rechenberg, M., Breukink, E., den Blaauwen, T., Gross, C. A. \& Vollmer, W. (2010). Cell, 143, 1097-1109.

Zeytuni, N., Ozyamak, E., Ben-Harush, K., Davidov, G., Levin, M., Gat, Y., Moyal, T., Brik, A., Komeili, A. \& Zarivach, R. (2011). Proc. Natl Acad. Sci. USA, 108, E480-E487. 